Portland State University

PDXScholar

7-1-2006

\title{
Measuring the Influence of Built Neighborhood Environments on Walking in Older Adults
}

\author{
Yvonne L. Michael \\ Oregon Health and Science University \\ Tracey Beard \\ Oregon Health \& Science University \\ Dongseok Choi \\ Oregon Health \& Science University \\ Stephanie Farquhar \\ Portland State University \\ Nichole Carlson
}

Follow this and additional works at: https://pdxscholar.library.pdx.edu/commhealth_fac

Part of the Community-Based Research Commons, and the Community Health and Preventive Medicine Commons

Let us know how access to this document benefits you.

\section{Citation Details}

Michael, Y., Beard, T., Choi, D., Farquhar, S., \& Carlson, N. (2006). Measuring the influence of built neighborhood environments on walking in older adults. Journal Of Aging And Physical Activity, 14(3), 302-312.

This Article is brought to you for free and open access. It has been accepted for inclusion in Community Health Faculty Publications and Presentations by an authorized administrator of PDXScholar. Please contact us if we can make this document more accessible: pdxscholar@pdx.edu. 


\title{
Measuring the Influence of Built Neighborhood Environments on Walking in Older Adults
}

\author{
Yvonne Michael, Tracey Beard, Dongseok Choi, \\ Stephanie Farquhar, and Nichole Carlson
}

\begin{abstract}
There is a need for greater understanding of how perceptions and objective measures of the physical environment influence physical activity among seniors. The goal of this study was to examine the degree of association between perceived and objective characteristics of the neighborhood environment and the relation of each type of measurement to neighborhood walking in older adults. Data on self-reported frequency of walking in the neighborhood and perceived measures of neighborhood environment from 105 older adults were linked to objective measures assessed by geographic information systems and an audit instrument. Perceived and objective measurements of the built environment exhibited a low degree of agreement (kappas: <.20). After adjustment for education, age, and gender, presence of a mall was positively associated with neighborhood walking in both the objective and perceived models.
\end{abstract}

Key Words: walking, measurement, environment design

Research strongly supports the benefits of physical activity in reducing adverse health outcomes and preventing obesity in older adults (U.S. Preventive Services Task Force, 1996). As Americans age, their activity levels decline, and by age 75, nearly a third of all men and half of all women do not participate in any form of physical activity (Centers for Disease Control and Prevention, 1999).

As obesity rates in the United States continue to rise and widespread adoption of an active lifestyle has yet to occur, public health advocates are looking beyond individual behavior-change strategies to examine the physical and social environments that might be influencing physical activity levels (Office of the Surgeon General, n.d.). Taking a more ecological approach, public health researchers are turning to the impact of the built environment, traditionally the focus of urban planning and transportation disciplines, to assess its influence on physical activity

Michael, Beard, Choi, and Carlson are with Public Health and Preventive Medicine, Oregon Health and Science University Portland, OR 97239. Farquhar is with the School of Community Health, Portland State University, Portland, OR. 
(Frank \& Engelke, 2001; Handy, Boarnet, Ewing, \& Killingsworth, 2002). Examining how the environment acts as a facilitator or barrier to physical activity might inform the design of policy interventions and influence urban designs that promote activity (Dannenberg et al., 2003). Features of the built environment that have been analyzed include land-development patterns, transportation systems, and microscale urban design (Booth, Owen, Bauman, Clavisi, \& Leslie, 2000; Brownson, Baker, Housemann, Brennan, \& Bacak, 2001; King et al., 2003; Moudon, Hess, Snyder, \& Stanilov, 1997; Pucher \& Clorer, 1998; Saelens, Sallis, Black, \& Chen, 2003; Troped et al., 2001). An important measurement issue in this field is the use of perceived versus objective measures of the built environment (Saelens, Sallis, \& Frank, 2003). In studying physical activity in a neighborhood, examining both the perceived and objective perspective becomes particularly interesting. Certain perceived environmental features might be related to physical activity because of the increased awareness and familiarity of the environment among the physically active compared with less active neighborhood members (Kirtland et al., 2003). Objective measurements might reduce some of the subjectivity of the measurements but also might eliminate the role of an individual's perception that intuitively could be related to higher neighborhood activity level. Research investigating perception versus more objective measurements in relation to physical activity is limited (King et al., 2000; Kirtland et al.; Troped et al.), and none is specific to physical activity among older adults.

In this cross-sectional analysis we address two specific aims: (a) to quantify the degree of association between perceived and objective characteristics of walking-supportive neighborhood environments for older adults and (b) to assess the association between both the perceived and objective environmental elements on neighborhood walking among older adults.

\section{Methods}

\section{Data Source}

In this study, we utilized a subset of baseline data from Senior Health and Physical Exercise (SHAPE; Fisher, Li, Michael, \& Cleveland, 2004), a randomized walking intervention involving 582 senior residents from 56 randomly selected neighborhoods in Portland, OR. Portland has a recognized neighborhood system made up of 95 neighborhood associations defined by street boundaries (Portland's Neighborhood Network; Office of Neighborhood Environment, n.d.). A detailed description of the study design and methods has been published elsewhere (Fisher $\& \mathrm{Li}, 2004)$. Briefly, all participants in the SHAPE study met the following selection criteria: being 65 years of age or older, not participating in any formal physical activity in the past 30 days, and being able to walk without an assistive device (such as a walker). Neighborhoods were randomly assigned to either a leader-guided neighborhood-walking condition $(n=28)$ or an education-only control condition $(n$ =28). Data on demographic information, physical activity, and the neighborhood environment were collected from the study participants using 30- to 40-min face-toface interviews. Intervention and control neighborhoods did not differ significantly in terms of sociodemographic or exercise characteristics at baseline (Fisher \& Li). 
For the purposes of this study, a subsample of 10 control neighborhoods was selected from the SHAPE study to examine the effects of the physical environment on walking among seniors (Cunningham, Michael, Farquhar, \& Lapidus, 2005). Neighborhoods were stratified by a "walking friendliness" ranking variable. This variable (with four categories: high, medium-high, medium-low, and low) was derived for each neighborhood, based on available social and environmental data that was hypothesized to correlate with walking and physical activity - high income, high senior population density, high proportion of White residents, low crime rates, and higher number of facilities for walking (e.g., parks, gardens). Ten neighborhoods were selected to represent a diverse subsample on the basis of "walkability": two high, two medium-high-, two medium-low-, and four low-walkability neighborhoods. Low-walkability neighborhoods were oversampled to ensure sufficient variability in observed and perceived problems across neighborhoods.

Baseline data from SHAPE participants living in these 10 SHAPE control neighborhoods $(N=105)$ were linked with objective measures of the neighborhood built environment assessed by geographic information systems (GIS) and an audit instrument.

\section{Measures}

Built-Environment Characteristics. Neighborhood-level built-environment characteristics included in this study were selected on the basis of prior research and included sidewalk quality, neighborhood graffiti and vandalism (aesthetics), and presence of shopping malls, parks, and trails (King et al., 2003; Kirtland et al., 2003; Sallis, Johnson, Calfas, Caparosa, \& Nichols, 1997; Troped et al., 2001). Each independent variable was assessed using objective and perceived measures (see Table 1). The objective measures were designed to conceptually match the questions about perceptions of the built environment included in the SHAPE survey. In most cases, the conceptual correspondence was high, for example, the measures assessing presence of sidewalks, sidewalk obstructions, malls, parks, and trails. To correspond with the perceived questions regarding whether graffiti and vandalism were neighborhood problems, we selected a measure that assessed the presence of "litter, graffiti, broken glass, etc." from the audit instrument. Both objective and perceived measures assessed visible evidence of disrepair that could reduce the aesthetic quality of the neighborhood.

Perceived Built-Environment Measures. Seniors' perceptions about their neighborhood environment were assessed in the SHAPE survey (Sallis et al., 1997). Specifically, seniors were asked to indicate "yes" or "no" if they had any of the following near their home: shopping mall, public park, or trails for walking, hiking, or running. Seniors were also asked how much they agreed or disagreed that certain issues were a problem in their neighborhood: "no sidewalks (or footpaths)," "unsafe sidewalks (obstacles to walking)," "graffiti," and "vandalism." A Likert scale ranging from 1 (strongly disagree) to 5 (strongly agree) was provided for their response.

Objective Built-Environment Measures. Data on sidewalk quality and neighborhood graffiti and vandalism were collected by trained research assistants using a reliable audit instrument specifically developed to examine the effects of the 
Table 1 Objective and Perceived Measures of Built Environment Variables

\begin{tabular}{|c|c|c|}
\hline Variable & $\begin{array}{l}\text { Objective: } \\
\text { RLIS/systematic } \\
\text { observation }\end{array}$ & $\begin{array}{l}\text { Perceived }{ }^{\mathrm{a}} \text { : } \\
\text { SHAPE }\end{array}$ \\
\hline & & $\begin{array}{l}\text { How much do you agree or } \\
\text { disagree that each of the } \\
\text { following things is a problem in } \\
\text { your neighborhood? Possible } \\
\text { responses } 1 \text { (strongly disagree) } \\
\text { to } 5 \text { (strongly agree) }\end{array}$ \\
\hline $\begin{array}{l}\text { Sidewalk } \\
\text { existence }\end{array}$ & $\begin{array}{l}\text { Systematic observation: } \\
\text { Are sidewalks continuous? } \\
\text { No }=0, \text { yes }=1 \\
\text { No sidewalks }=98\end{array}$ & $\begin{array}{l}\text { No sidewalks (or footpaths) are a } \\
\text { problem. }\end{array}$ \\
\hline $\begin{array}{l}\text { Sidewalk } \\
\text { obstructions }\end{array}$ & $\begin{array}{l}\text { Systematic observation: } \\
\text { Mark all that create } \\
\text { considerable obstruction/ } \\
\text { danger to pedestrian traffic. }\end{array}$ & $\begin{array}{l}\text { Unsafe sidewalks (obstacles to } \\
\text { walking) are a problem }\end{array}$ \\
\hline $\begin{array}{l}\text { Graffiti and } \\
\text { vandalism }\end{array}$ & $\begin{array}{l}\text { Systematic observation: } \\
\text { Any litter, graffiti, broken } \\
\text { glass, etc.? } 0=\text { none or } \\
\text { almost none. } 1=\text { yes, but } \\
\text { not dominant feature. } \\
2=\text { yes, dominant feature. }\end{array}$ & $\begin{array}{l}\text { Please circle YES or NO if you } \\
\text { have any of the following near } \\
\text { your home: }\end{array}$ \\
\hline $\begin{array}{l}\text { Presence of } \\
\text { shopping mall }\end{array}$ & RLIS & Shopping mall \\
\hline Presence of park ${ }^{\mathrm{b}}$ & $\mathrm{n} / \mathrm{a}$ & Public park \\
\hline Presence of trails & RLIS & $\begin{array}{l}\text { Trails for walking, hiking, } \\
\text { or running }\end{array}$ \\
\hline
\end{tabular}

Note . RLIS = Regional Land Information System; SHAPE = Senior Health and Physical Exercise intervention.

aPerceived variables were transformed into dichotomous variables. Responses of "strongly disagree," "disagree," or "neutral" for the statements on graffiti, vandalism, and sidewalk obstacles indicated that the features were not a problem (i.e., not present) and were coded as 0 . Responses of "agree" or "strongly agree" indicated that the problem was present and were coded as 1. For sidewalk existence, reverse coding was used.

${ }^{\mathrm{b}}$ There was no variability in the objective measure of parks among study neighborhoods, so it was dropped in subsequent analysis.

physical environment on walking among seniors in Portland, OR (Cunningham et al., 2005). The instrument incorporated items pertinent to seniors identified in a thorough review of urban-planning and health literature (Cunningham \& Michael, 2004). A full copy of the audit instrument can be obtained from the corresponding author (michaely@ohsu.edu). 
Individual SHAPE households were not mapped in this study because of the potential loss of confidentiality. Instead, all street segments in the 10 study neighborhoods were enumerated using GIS mapping, and a 10\% sample of street segments was randomly selected to represent the general characteristics of the neighborhood. Segments representing industrial blocks or freeways were excluded. Neighborhood-level measures of the built-environment characteristics of interest were created by aggregating up objective assessment data from the randomly selected segments for each of the 10 neighborhoods.

Five teams of two trained research assistants observed 355 neighborhood segments from November 2002 through August 2003. Data were collected for both sides of the street. On average, administration of the audit required 17 min per completed segment. Tests of interrater agreement for the audit instrument found that raters were in good to excellent agreement on most of the items (Cunningham et al., 2005). For the purposes of this report, data from the audit on presence of sidewalk, identification of sidewalk obstructions, and presence of graffiti (and other evidence of vandalism) were used. Specifically, we observed $94 \%$ agreement between raters on presence of sidewalk, identification of sidewalk obstructions ranged from $72 \%$ to $94 \%$ agreement, and there was $72 \%$ agreement on presence of graffiti. Additional details on the development and testing of the instrument are provided elsewhere (Cunningham et al.).

A GIS database, Regional Land Information System (RLIS), provided objective neighborhood data on presence of malls, parks, and trails. RLIS uses data derived from assessment and taxation records.

Neighborhood Walking. By selecting walking in the neighborhood as our measure of physical activity, we focused on activity in the area described by the builtenvironment measures. Neighborhood walking was measured using the response to the following question: "Over the past 12 months, how much have you done the following? Walked or strolled in the neighborhood." Possible responses were anchored on a 5-point Likert scale from 1 (not at all) to 5 (a great deal). Participants who responded "not at all," "a little bit," or "a moderate amount" were classified as low walkers. Participants responding "quite a bit" or "a great deal" were classified as high walkers.

\section{Statistical Analysis}

Neighborhood walking and other characteristics of the study population were evaluated using cross-tabulation and chi-squared statistics. Percentage agreement between the self-reported and objectively derived measures of neighborhood environment was calculated. The relation between the measures was tested with the kappa statistic (Rosner, 2000).

Separate univariate logistic-regression models were built to explore the association between neighborhood walking and (a) the perceived environment measures and (b) the objective environment measures. All variables with a $p$ value of .25 in the univariate analysis were selected for the multivariate model (Mickey \& Greenland, 1989). Multivariate logistic-regression models were employed to explore the association adjusted for other demographic and socioeconomic-status variables such as age, race, gender, education, and income. Variables with large $p$ values $(>.5)$ were removed from the multivariate model, and the likelihood-ratio 
test was used to determine whether these variables could be permanently eliminated from the model.

Linearity was assessed for continuous variables in the main effects model by categorizing these variables into quartiles and attempting several transformations of the variable. Improvement in model fit was determined by an increase in overall model significance and corresponding significant decrease in deviance. Final models were selected based on the Hosmer and Lemeshow goodness-of-fit test.

\section{Results}

Neighborhoods included in this analysis had median property values ranging from $\$ 107,430$ to $\$ 224,780$. The percentage of people 65 years of age and older living in each neighborhood ranged from 4.9 to 18.9. The mean age of the older adults included in this study was 75.1 (sample $S D=6.29$ ), and the range spanned from 65 to 92 . Approximately $67 \%$ of these older adults were women. The study sample was primarily Caucasian (90\%), but participants were diverse in terms of education and income. Ten percent of participants had a household income of less than $\$ 10,000$, and $30 \%$ had an income above $\$ 30,000$. Only $15 \%$ did not have a high school degree. Thirty percent of participants reported some college education, and $16 \%$ reported having a graduate degree. According to 2000 census data, $11.6 \%$ of people living in Portland were 65 years of age or older, and median property value was $\$ 157,900$.

\section{Neighborhood Walking}

Nearly $30 \%$ of participants reported that they walked or strolled in the "neighborhood a great deal," and less than $12 \%$ reported not walking at all in the neighborhood. Forty-seven participants were classified as high walkers (reported walking quite a bit or a great deal), and 58 participants were categorized as low (or inactive) neighborhood walkers. Participants who reported high neighborhood walking tended to be younger $(p=.03)$ and were more likely to be White ( $p=$ $.01)$. No significant differences were observed between participants that reported high neighborhood walking and low neighborhood walking in terms of gender, education, or income.

\section{Degree of Agreement Between Objective and Perceived Measures}

The kappa values between perceived and objective measurements were low for all variables, ranging from -.07 to .20 (Table 2), indicating a low degree of agreement and reproducibility between dichotomous perceived and objective measurements of trails, graffiti and vandalism, sidewalk existence, and sidewalk obstruction (Table 2). As would be expected in urban neighborhoods, only 10 of the older adults lived in a neighborhood with a shopping mall near their home, and all correctly identified the presence of the mall. Older adults were more likely to report sidewalk obstructions in neighborhoods where objective observers noted no obstructions. Parks were present in every neighborhood based on the objective data, and presence of parks was confirmed by $90 \%$ of the older adults. 
Table 2 Agreement Between Objective and Perceived Measures of Neighborhood Environment

\begin{tabular}{|c|c|c|c|c|c|c|}
\hline & $Y_{\text {obj }} / Y_{\text {per }}$ & $Y_{\text {obj }} / N_{p e r}$ & $N_{\text {obj }} / Y_{\text {per }}$ & $\mathbf{N}_{\text {obj }} / \mathbf{N}_{\text {per }}$ & kappa & $\begin{array}{c}\% \\
\text { Agreement }\end{array}$ \\
\hline $\begin{array}{l}\text { Graffiti and } \\
\text { vandalism }\end{array}$ & 19 & 21 & 21 & 25 & .018 & 51 \\
\hline $\begin{array}{l}\text { Sidewalk } \\
\text { obstruction }\end{array}$ & 50 & 15 & 16 & 4 & -.031 & 64 \\
\hline Parks & 85 & 9 & 0 & 0 & $\mathrm{n} / \mathrm{a}$ & 90 \\
\hline Malls & 10 & 0 & 39 & 44 & .195 & 58 \\
\hline Trails & 20 & 20 & 28 & 21 & -.07 & 46 \\
\hline \multicolumn{7}{|c|}{$\begin{array}{l}\text { Note. } \mathrm{Y}_{\text {obj }} / \mathrm{Y}_{\text {per }}=\text { number of responses that were positive for objectively measured neighborhood } \\
\text { environment and positive for perceived measures of neighborhood environment; } \mathrm{Y}_{\text {obj }} / \mathrm{N}_{\text {per }}=\text { number } \\
\text { of responses that were positive for objectively measured neighborhood environment and negative for } \\
\text { perceived measures of neighborhood environment; } \mathrm{N}_{\text {obj }} / \mathrm{Y}_{\text {per }}=\text { number of responses that were negative } \\
\text { for objectively measured neighborhood environment and positive for perceived measures of neigh- } \\
\text { borhood environment; } \mathrm{N}_{\text {obj }} / \mathrm{N}_{\text {per }}=\text { number of responses that were negative for objectively measures } \\
\text { neighborhood environment and negative for perceived measures of neighborhood environment. }\end{array}$} \\
\hline
\end{tabular}

\section{Association Between Neighborhood Built Environment and Walking}

The remaining analysis was performed for White, non-Hispanic participants because race modified the relation between built environment and neighborhood walking, but insufficient numbers of non-White study participants prohibited stratified analyses. In the univariate analysis of objective neighborhood characteristics with neighborhood walking, presence of a mall was positively associated with neighborhood walking $(\mathrm{OR}=4.72, p=.035)$, and the presence of graffiti and vandalism was negatively associated with neighborhood walking $(\mathrm{OR}=0.58, p=.204)$. No other objective measures approached statistical significance. Among perceived neighborhood characteristics, presence of a mall and trails were positively associated with neighborhood walking $(\mathrm{OR}=1.75, p=.178$, and $\mathrm{OR}=1.64, p=.245)$. No other perceived neighborhood characteristics approached statistical significance. After adjustment for education, age, and gender, presence of a mall remained in both the objective and perceived models of walking: OR $=4.12(p=.147)$ and $\mathrm{OR}=2.10(p=.108)$, respectively. Graffiti and vandalism was also retained in the multivariate objective model ( $\mathrm{OR}=0.57, p=.28)$. No other perceived neighborhood-environment variables were retained in the multivariate model. The Hosmer and Lemeshow goodness-of-fit $p$ values were .256 and .762 for the objective and perceived models, respectively, indicating that the models fit the data well. 


\section{Discussion and Conclusions}

Consistent with previous research with older adults (King et al., 2003), this analysis confirms that availability of utilitarian destinations (represented in this study by shopping malls) is positively associated with walking. Of course, causality cannot be inferred from this cross-sectional study.

We found little agreement between the objective and perceived measures of the built-environment features. This might reflect conceptual differences between our perceived and objective measures (for example, the differences in the objective and perceived questions related to aesthetics). Kirtland and colleagues (2003), however, also reported fair to low agreement between neighborhood items comparing survey responses to the GIS objective measures $(\kappa=-.02$ to .37$)$. Future research on the relationship between neighborhood and physical activity in older adults should include objective, as well as perceived, measures of built-environment characteristics in the design phase to further evaluate differences in findings related to the different measurement methods.

Walking is the most common form of physical activity in older adults (Centers for Disease Control and Prevention, 1999). Furthermore, those who walk for exercise in the United States most frequently report walking on neighborhood streets for this purpose (Eyler, Brownson, Bacak, \& Housemann, 2003). The present study confirmed the results of prior research indicating that sociodemographic variables such as race and age are associated with physical activity (DiPietro, 2001). Recent estimates of the percentage of older adults engaged in walking from U.S. populationbased samples vary from $47 \%$ to $70 \%$ (Lee, 2005). Only $12 \%$ of our study sample reported not walking at all in the neighborhood. The national and state data include those who cannot walk without an assistive device (a group that would probably be more likely to not walk) and thus would be expected to have a higher percentage of nonwalkers than our study sample because all participants in our study sample met the criteria of being able to walk without an assistive device.

Major limitations of this study include the small sample size and homogeneous study population. Despite including more neighborhoods with "low walkability" characteristics a priori, we observed limited variability in the built-environment characteristics of study neighborhoods (e.g., parks), which limited our ability to observe significant associations between some neighborhood characteristics and walking. The results might not be generalizable to older adults other than those living in urban areas in the Northwest.

Limitations of GIS information in studies of this kind should be noted (Melnick \& Fleming, 1999). Because neighborhood size differed greatly in this study (from 119 acres in the smallest neighborhood to 7,055 in the largest one), GIS data on the built environment might not be representative of the area in which a participant lived. Similarly, participants were not asked to define what they considered to be their neighborhood, and the GIS boundaries of neighborhood might not match a person's perceived neighborhood area. In addition, the GIS database available for the Portland neighborhoods does not include information on the presence of graffiti and vandalism, sidewalk presence, and sidewalk obstructions. However, audit assessments used in this study provided measures of these items with high internal consistency. 
It is possible that for these older neighborhood residents, perceptions of neighborhood boundary might be inconsistent with the actual street-defined neighborhood boundary used in this study. To address this issue, future studies could work with older residents early in the project to generate an agreed-on neighborhood boundary or definition. For example, a project could hold a few focus groups and ask residents how they would spatially define their neighborhood boundaries. This might include street names and distances but will likely also include structures (e.g., churches and grocery stores). An alternative approach involving less participation and buy-in could be to operationalize "local neighborhood" as the quarter-mile buffer around a person's residence when analyzing GIS data and within a 5-min walk when collecting perceived-neighborhood data in order to focus on the geographic community most proximal to each individual (Congress for the New Urbanism, n.d.).

In this study, only six features of the built environment were examined in association with neighborhood walking in older adults. Research should be conducted that expands on the features and destinations of this study, including both positive and negative characteristics to determine whether other components are related to increased walking in this population.

\section{Acknowledgments}

This research was supported by National Institutes of Health/National Institute of Aging [AG022240] and the Borchard Center Foundation for Law and Aging.

The authors would like to thank John Fisher, who contributed the SHAPE data for this analysis, and Irina Sharkova, who provided the GIS data. A special note of thanks to Hannah Kellogg, Mandy Green, Ruth Rowland, Grazia Cunningham, Meredith Davis, Elizabeth Goodman, Katherine Ona, Setiawati Rahardjo, Lea Sturges, and Linda Soto, who collected data for this project.

\section{References}

Booth, M.L., Owen, N., Bauman, A., Clavisi, O., \& Leslie, E. (2000). Social-cognitive and perceived environment influences associated with physical activity in older Australians. Preventive Medicine, 31(1), 15-22.

Brownson, R.C., Baker, E.A., Housemann, R.A., Brennan, L.K., \& Bacak, S.J. (2001). Environmental and policy determinants of physical activity in the United States. American Journal of Public Health, 91(12), 1995-2003.

Centers for Disease Control and Prevention. (1999). Physical activity and health: Older adults. Retrieved May 9, 2006, from http://www.cdc.gov/nccdphp/sgr/olderad.htm

Congress for the New Urbanism. (n.d.). Congress for the new urbanism: Tour. Retrieved November 2, 2005, from http://www.cnu.org/about/index.cfm?formaction = tour \&CFID $=12066179 \& \mathrm{CFTO}$

Cunningham, G., \& Michael, Y. (2004). Concepts guiding the study of the impact of the built environment on physical activity for older adults: A review of the literature. American Journal of Health Promotion, 18(6), 435-443.

Cunningham, G., Michael, Y., Farquhar, S., \& Lapidus, J. (2005). Developing a reliable senior walking environmental assessment tool. American Journal of Preventive Medicine, 29(3), 215-217.

Dannenberg, A.L., Jackson, R.J., Frumkin, H., Schieber, R.A., Pratt, M., Kochtitzky, C., et al. (2003). The impact of community design and land-use choices on public health: A scientific research agenda. American Journal of Public Health, 93(9), 1500-1508. 
DiPietro, L. (2001). Physical activity in aging: Changes in patterns and their relationship to health and function. Journals of Gerontology Series A: Biological Sciences and Medical Sciences, 56(2), 13-22.

Eyler, A.A., Brownson, R.C., Bacak, S.J., \& Housemann, R.A. (2003). The epidemiology of walking for physical activity in the United States. Medicine and Science in Sports and Exercise, 35(9), 1529-1536.

Fisher, K.J., \& Li, F. (2004). A community-based walking trial to improve neighborhood quality of life in older adults: A multilevel analysis. Annals of Behavioral Medicine, 28(3), 186-194.

Fisher, K.J., Li, F., Michael, Y., \& Cleveland, M. (2004). Neighborhood-level influences on physical activity among older adults: A multilevel analysis. Journal of Aging and Physical Activity, 12(1), 45-63.

Frank, L., \& Engelke, P. (2001). The built environment and human activity patterns: Exploring the impact of urban form on public health. Journal of Planning Literature, 16(2), 202-218.

Handy, S.L., Boarnet, M.G., Ewing, R., \& Killingsworth, R.E. (2002). How the built environment affects physical activity: Views from urban planning. American Journal of Preventive Medicine, 23(2 Suppl.), 64-73.

King, A.C., Castro, C., Wilcox, S., Eyler, A.A., Sallis, J.F., \& Brownson, R.C. (2000). Personal and environmental factors associated with physical inactivity among different racial-ethnic groups of U.S. middle-aged and older-aged women. Health Psychology, 19(4), 354-364.

King, W.C., Brach, J.S., Belle, S., Killingsworth, R., Fenton, M., \& Kriska, A.M. (2003). The relationship between convenience of destinations and walking levels in older women. American Journal of Health Promotion, 18(1), 74-82.

Kirtland, K.A., Porter, D.E., Addy, C.L., Neet, M.J., Williams, J.E., Sharpe, P.A., et al. (2003). Environmental measures of physical activity supports: Perception versus reality. American Journal of Preventive Medicine, 24(4), 323-331.

Lee, Y.S. (2005). Gender differences in physical activity and walking among older adults. Journal of Women and Aging, 17(1-2), 55-70.

Melnick, A., \& Fleming, D. (1999). Modern geographic information system-Promise and pitfalls. Journal of Public Health Management Practice, 5, viii-x.

Mickey, R.M., \& Greenland, S. (1989). The impact of confounder selection criteria on effect estimation. American Journal of Epidemiology, 129(1), 125-137.

Moudon, A., Hess, P., Snyder, M., \& Stanilov, K. (1997). Effects of site design on pedestrian travel in mixed use, medium-density environments. Transportation Research Record, 1578, 48-55.

Office of the Surgeon General. (n.d.). Overweight and obesity: What you can do. Retrieved May 9, 2006, from http://www.surgeongeneral.gov/topics/obesity/calltoaction/fact_ whatcanyoudo.htm

Portland's Neighborhood Network, Office of Neighborhood Environment. (n.d.). Retrieved January 28, 2005, from http://www.portlandonline.com/oni/index.cfm?\&a $=22827 \& \mathrm{c}$ $=29020$

Pucher, J., \& Clorer, S. (1998). Urban transportation in Germany: Providing feasible alternatives to the car. Transport Reviews, 18, 285-310.

Rosner, B. (2000). Fundamentals of biostatistics (5th ed.). Belmont, CA: Duxbury Thomas Learning Co.

Saelens, B.E., Sallis, J.F., Black, J.B., \& Chen, D. (2003). Neighborhood-based differences in physical activity: An environment scale evaluation. American Journal of Public Health, 93(9), 1552-1558.

Saelens, B.E., Sallis, J.F., \& Frank, L.D. (2003). Environmental correlates of walking and cycling: Findings from the transportation, urban design, and planning literatures. Annals of Behavioral Medicine, 25(2), 80-91. 
Sallis, J.F., Johnson, M.F., Calfas, K.J., Caparosa, S., \& Nichols, J.F. (1997). Assessing perceived physical environmental variables that may influence physical activity. Research Quarterly for Exercise and Sport, 68, 345-351.

Troped, P.J., Saunders, R.P., Pate, R.R., Reininger, B., Ureda, J.R., \& Thompson, S.J. (2001). Associations between self-reported and objective physical environmental factors and use of a community rail-trail. Preventive Medicine, 32(2), 191-200.

U.S. Preventive Services Task Force. (1996). Guide to clinical preventive services (2nd ed.). Baltimore, MD: Williams \& Wilkins. 